\title{
Elevated level of some chemokines in plasma of gastric cancer patients
}

\author{
MONIKA BAJ-KRZYWORZEKA ${ }^{l}$, KAZIMIERZ WEGGARCZYK', JAREK BARAN ${ }^{1}$, \\ ANTONI SZCZEPANIK', MIROSŁAW SZURA ${ }^{2}$, MACIEJ SIEDLAR ${ }^{l}$ \\ ${ }^{1}$ Department of Clinical Immunology, Institute of Paediatrics, Jagiellonian University Medical College, Krakow, Poland \\ ${ }^{2}$ First Department of General and Gastrointestinal Surgery, Jagiellonian University Medical College, Krakow, Poland
}

\begin{abstract}
Introduction: Gastric cancer is one of the most common cancer-related causes of death. This is mainly due to the lack of good noninvasive method/biomarkers suitable for early-tumour diagnosis and planning of further therapy modalities. Chemokines play an important role in cancer progression and metastasis formation. In gastric cancer patients, clinical relevance of CXCL12 and CCL5 level has been postulated.

Aim of the study: Efforts were undertaken to examine whether expanded chemokine range may be relevant for evaluation of preoperative staging of gastric cancer patients.

Material and methods: Plasma from 66 gastric cancer patients and 11 healthy controls was obtained, and CCL2, CCL3, CCL4, CCL5, CXCL8, CXCL9, and CXCL10 levels were determined by flow cytometry FlexSet system.

Results: In gastric cancer patients' plasma an increased level of CCL2, CCL4, CCL5, CXCL8, CXCL9, and CXCL10 was observed. In the case of CCL2, CXCL9, and CXCL10, the chemokine levels correlated with advanced (III and IV in TNM classification) disease stage. In the case of CCL4, CCL5, and CXCL8, elevated levels were observed in all cancer patients in comparison to healthy donors.

Conclusions: The accuracy of preoperative diagnosis in gastric cancer may include the monitoring of a wide range of chemokines in patients' plasma. Increased levels of chemokines may warn that the disease is more advanced than conventional diagnostic procedures suggest.
\end{abstract}

Key words: gastric, cancer, chemokines.

(Cent Eur J Immunol 2016; 41 (4): 358-362)

\section{Introduction}

Chemokines are a family of small ( $8-14 \mathrm{kDa})$ cytokines that induce migration of various cell types $[1,2]$. They mediate the biological effect on target cells through widely distributed G-protein-coupled receptors, which are structurally characterised by seven transmembrane spanning domains. Chemokines regulate infiltration of leukocytes to the sites of inflammation, injury, or tumour bed $[1,2]$. The availability of chemokines in the site of inflammation is regulated at the level of their biosynthesis, by proteolytic processing, and by mobilisation of the chemokines by cell surface molecules. Chemokines are produced by many cell types in the tumour microenvironment including leukocytes, endothelial cells, fibroblasts, epithelial cells, and cancer cells themselves [3]. In the tumour bed, chemokines from CXCL (CXCL1, 2, 3, 6, 8, 12) and CCL (CCL2, 5, 7 ) families were previously detected $[4,6]$. As a conse- quence, chemokines are also present in cancer patients' blood.

The biological role of chemokines in tumour progression is important because they promote tumourigenesis and metastasis formation [2]. Chemokines are responsible for modulation of tumour growth by regulation of angiogenesis (e.g. secretion of metalloproteinases [MMP] [4-6]), tumour-specific immune response (e.g. polarisation of tumour-associated macrophages (TAMs) [2, 4]), and stimulation of tumour cell proliferation [7] in an autocrine or paracrine fashion [8].

Gastric cancer is the fourth most common cancer worldwide and one of the major health problem [9]. Surgical resection is still a primary treatment option, although overall survival outcomes remain unsatisfactory. Numerous studies were undertaken to find specific "markers" of gastric cancer progression to improve diagnostic procedure. The most promising data suggests that the production of SDF-1 $\alpha$ in the primary cancer may be such a prognostic

1. Correspondence: Monika Baj-Krzyworzeka, Department of Clinical Immunology, Institute of Paediatrics, Jagiellonian University Medical College, Wielicka 265, 30-663 Krakow, Poland, e-mail: mibaj@cyf-kr.edu.pl Submitted: 28.09.2016; Accepted: 10.10.2016 
factor [10]. High CXCR4/high SDF-1 $\alpha$ (CXCL12) expression in tumour cells is significantly associated with depth of cancer invasion, lymph node involvement, and more advanced stage of disease [10]. Also, elevated level of RANTES (CCL5) in serum of gastric cancer patients correlates with poor prognosis [11]. CCL5 is also a candidate biomarker because its measurement is a noninvasive method useful for diagnosis of gastric adenocarcinoma [12].

\section{Aim of the study}

The aim of the study was to determine if other chemokines, namely MCP-1 (CCL2), MIP-1 $\alpha$ (CCL3), MIP$1 \beta$ (CCL4), IL-8 (CXCL8), MIG (CXCL9), and IP-10 (CXCL10), are present in the plasma of gastric cancer patients (stage I-IV TNM), and if they might be useful as serum/plasma biomarkers to improve accuracy of preoperative staging.

\section{Material and methods}

\section{Patients and healthy donors}

Sixty-six consecutive patients (median age 66 years, range 40-76 years) with biopsy-proven gastric cancer at different clinical stages were studied (Table 1). All patients underwent a surgical treatment, and the pathological staging was completed according to UICC TNM $7^{\text {th }}$ edition classification. In all patients with the fourth-stage palliative resection was performed. None of the patients received preoperative chemotherapy or chemoradiotherapy. No concomitant active inflammatory diseases were diagnosed. There were no statistically significant differences between patients in stages I+II vs. III+IV regarding age, sex, and tumour type according to the Lauren classification.

The control group consisted of 11 healthy donors (median age 41 years, range 33-61 years). All study participants signed an informed consent form, and the Bioethical Committee of the Jagiellonian University Medical College (KBET/491/B/2003) approved the study protocol.

\section{Determination of chemokine levels}

Plasma from gastric cancer patients was collected preoperatively, and the concentrations of chemokines CCL2, CCL3, CCL4, CCL5, CXCL8, CXCL9, and CXCL10 were measured simultaneously using a Flex Set bead array (BD Biosciences, Immunocytometry Systems, San Jose, CA) followed by flow cytometric analysis (FACSCanto, BD Biosciences), as described previously [13]. The Flex Set beads were discriminated in FL-4 and FL-5 fluorescence channels, while the concentration of specified chemokine was determined by the intensity of FL-2 fluorescence. The amount of chemokines was computed by using the respective standard reference curve and FCAP Array software
(BD Biosciences). For all chemokines the detection level was $10 \mathrm{pg} / \mathrm{ml}$.

\section{Statistical analysis}

Statistical analysis was performed by nonparametric Mann-Whitney test. Differences were considered significant at $p$-values $<0.05$.

\section{Results}

By the use of bead array assay we detected significantly elevated levels of specified chemokines (CCL2, CCL4, CCL5, CXCL8, CXCL9, and CXCL10; Fig. 1A-F) in the plasma of gastric cancer patients, compared to healthy donors.

In our study, the levels of CCL2, CXCL9, and CCL10 correlated with clinical stage of disease, being highest in more advanced cancer patients (III and IV stage in TNM classification Fig. 1A, E, F). The levels of CCL4 and CCL5 were markedly elevated in the patients' plasma despite their clinical stage (Fig. 1B, C). The level of CXCL8 was significantly elevated in the plasma of patients with II-IV disease stage (Fig. 1D). The level of CCL2 was elevated only in plasma of patients at stage IV (Fig. 1A). In case of CCL3, its level was very low and we did not detect any differences between patients and healthy donors (data not shown). Chemokine levels did not correlate with histological type of cancer, but with the TNM stage.

\section{Discussion}

The presented study shows that the level of several chemokines in the plasma of gastric cancer patients is elevated when compared to healthy donors. The differences in chemokine levels are related to cancer staging (TNM classification) rather than histological type of tumour (no differences between LI and LII in Lauren classification, data not shown). The elevated level was detected, not only in relation to well documented CCL5 and CXCL12 [11, $14,15]$, but also to other chemokines from CC (CCL2, CCL4) and CXC (CXCL8, CXCL9, CXCL10) families.

In our study we observed an increase of CCL5 in plasma of gastric cancer patients, despite their clinical stage. Elevated levels of CCL5 were previously described by other authors $[11,12]$. This was also the case in patients

Table 1. Patients' characteristics

\begin{tabular}{lc}
\hline Age & $66(40-76) \pm 9.8$ \\
\hline Sex & $\mathrm{K}-25 / \mathrm{M}-41$ \\
\hline Stage I/II/III/IV & $\mathrm{I}-11, \mathrm{II}-11, \mathrm{III}-22$, IV -22 \\
\hline Lauren I/II/III & $\mathrm{I}-30 /$ II -32 / III -4 \\
\hline Total gastrectomy & 56 \\
\hline Subtotal gastrectomy & 10 \\
\hline
\end{tabular}



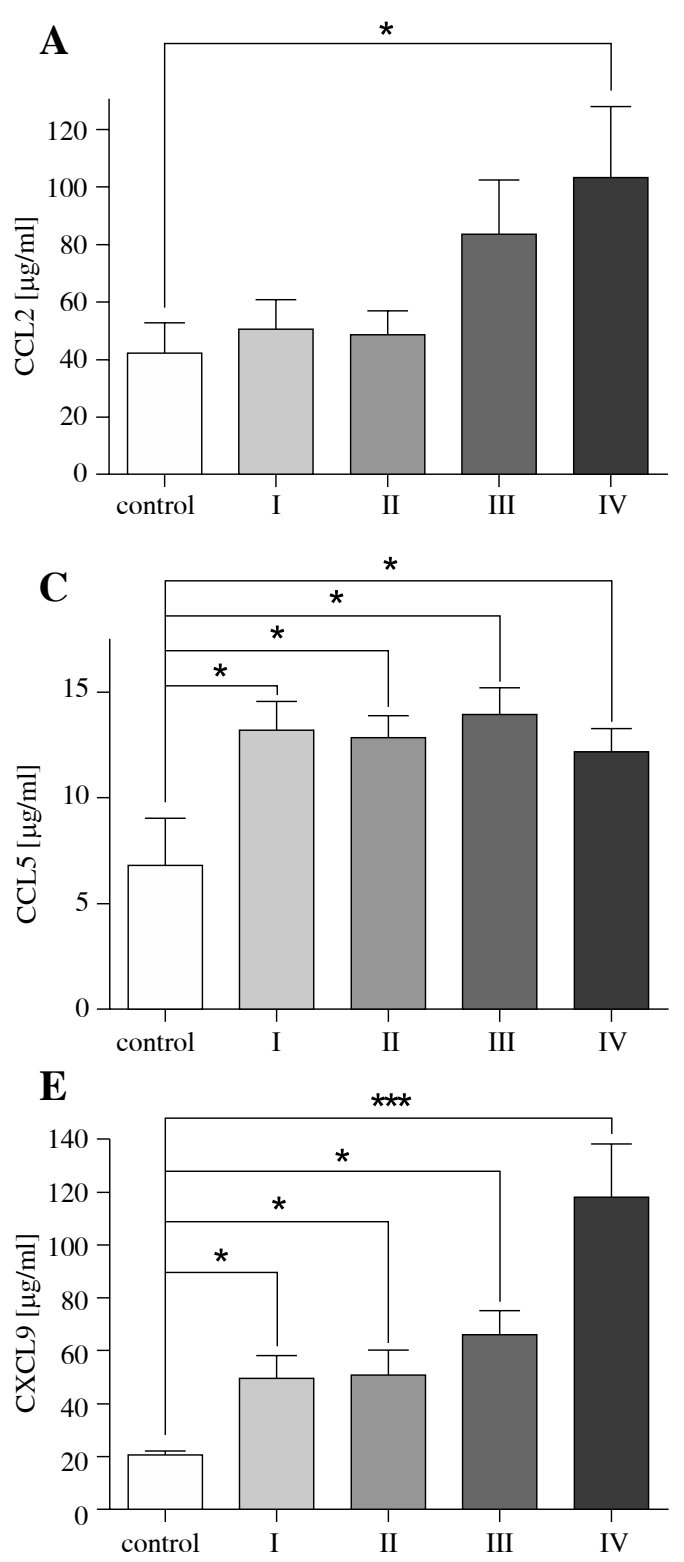
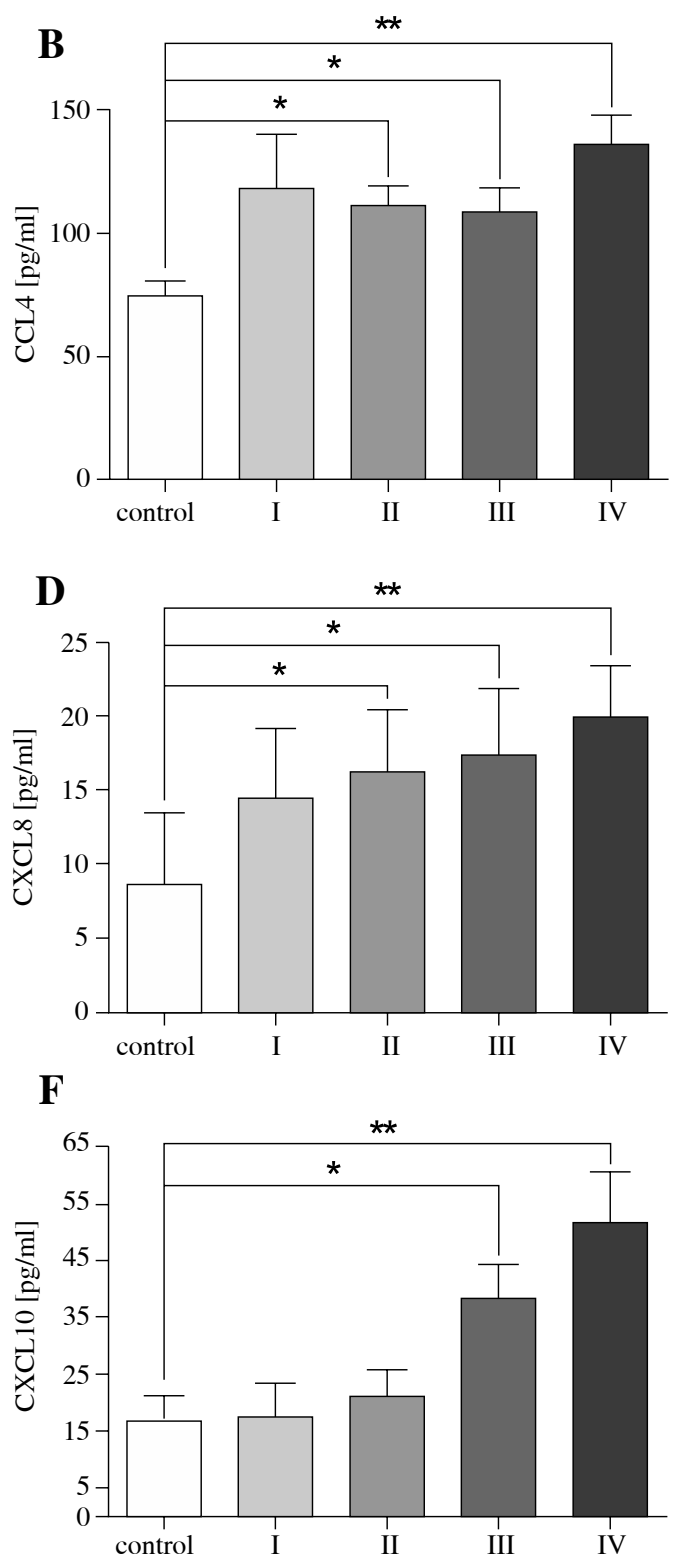

$* p<0.05, * * p<0.001$ vs. control

Fig. 1. The concentration of chemokines: A) CCL2, B) CCL4, C) CCL5, D) CXCL8, E) CXCL9, F) CXCL10) in plasma from gastric cancer patients (stages are labelled below) and healthy donors (control) determined by FlexSet method. Data are presented as mean $\pm \mathrm{SD}$

with breast cancer [16], where levels of CCL5 and CCL2 correlated with tumour progression [16]. In our study, CCL2 levels were increased, particularly in plasma of patients at stage IV. This corroborates with data published by Jianghong et al. [17] indicating that CCL2 may have a predictive value in such a group of patients. CCL2 has proangiogenic activity, and this activity results from macrophage recruitment and activation [18].
Increased levels of CCL3 in the plasma of gastric cancer patients was described for the first time by Rajkumar $e t$ al. as being involved in gastric cancer tumorigenesis [20]. However, in our data the CCL3 level was low and we did not observe differences between healthy donors and cancer patients. The discrepancies may result from the heterogeneity of the Rajkumar study group reflected in the wide range of CCL3 concentrations. 
CCL4 was previously detected in gastric tumour cell cytoplasm (intracellular form). Its expression seems to be dependent on the tumour type (higher expression in non-solid type of poorly differentiated carcinoma) [19]. Herein we have shown that CCL4 is present in the plasma of gastric cancer patients, and its level is significantly elevated compared to healthy donors. This is in line with the data published by Rajkumar et al. [20].

Gastric cancer cells may produce CXCL8 both in vitro and in vivo [5, 21]. We observed elevated levels of CXCL8 in the plasma of all gastric cancer patients, which correlated with the progression of the disease. CXCL8 was previously identified as a strong angiogenic factor in lung, ovarian, prostate, and gastric cancer [22]. CXCL8 may also facilitate tumour growth by inducing chemotaxis of neutrophilic granulocytes, which may further promote angiogenesis, tumour growth, and metastasis formation by releasing metalloproteases (MMPs) and vascular endothelial growth factor (VEGF) [23].

The production of CXCL9, CXCL10, and CXCL11 is induced by other cytokines, including members of the interleukin family and interferons. These chemokines inhibit angiogenesis in colon carcinoma, melanoma, and uterine cervical cancers and are considered as angiostatic chemokines [22]. High levels of CXCL9 and CXCL10 were detected previously by immunohistochemistry in lymphocyte-rich gastric cancers, characterised by increased levels of lymphatic invasion and unfavourable prognosis [24]. CXCL10 and CXCL9 were expressed by peritumorous macrophages, which attract $\mathrm{T}$ cells [25]. Interestingly, plasma levels of CXCL9 and CXCL10 decreased significantly in gastric cancer patients after surgery [20].

In summary, the current state of knowledge leads to the conclusion that increased levels of chemokines in plasma may help tumour cells to migrate and invade. In this report we are trying to draw attention to a broad range of chemokines, the levels of which are simultaneously elevated in gastric cancer patients and, in consequence, may serve as adequate indicators of disease stage. Preoperative testing of chemokine levels may help to predict the advance of tumours, including the "biological" potential, which reflects the TNM classification.

This study was supported by the State Committee for Scientific Research (2 PO5A 049 29).

The authors declare no conflict of interest.

\section{References}

1. Rossi D, Zlotnik A (2000): The biology of chemokines and their receptors. Annu Rev Immunol 18: 217-242.

2. Balkwill F (2003): Chemokine biology in cancer. Semin Immunol 1: 49-55.
3. Le Y, Zhou Y, Iribarren P, Wang J (2004): Chemokines and chemokine receptors: their manifold roles in homeostasis and disease. Cell Mol Immunol 1: 95-104.

4. Mantovani A, Sozzani S, Locati M, et al. (2002): Macrophage polarization: tumour-associated macrophages as a paradigm for polarized M2 mononuclear phagocytes. Trends Immunol 23: 549-555.

5. Kido S, Kitadai Y, Hattori N, et al. (2001): Interleukin 8 and vascular endothelial growth factor- prognostic factors in human gastric carcinomas? Eur J Cancer 37: 1482-1487.

6. Strieter RM, Belperio JA, Burdick MD, et al. (2004): CXC chemokines: angiogenesis, immunoangiostasis and metastasis in lung cancer. Ann N Y Acad Sci 1028: 351-360.

7. Zhu YM, Webster SJ, Flower D, Woll PJ (2004): Interleukin-8/CXCL8 is a growth factor for human lung cancer cells. Br J Cancer 91: 1970-1976.

8. Raman D, Baugher PJ, Thu YM, Richmond A (2007): Role of chemokines in tumour growth. Cancer Lett 256: 137-165.

9. Lee HJ, Kim SW, Kim HY, et al. (2009): Chemokine receptor CXCR4 expression, function and clinical implications in gastric cancer. Int J Oncol 34: 473-480.

10. Lee HJ, Huang SM, Kim HY, et al. (2011): Evaluation of the combined expression of chemokine SDF-1a and its receptor CXCR4 as a prognostic marker for gastric cancer. Exp Ther Med 3: 499-504.

11. Kim HK, Song KS, Park YS, et al. (2003): Elevated levels of circulating platelet microparticles, VEGF, IL-6 and RANTES in patients with gastric cancer: possible role of a metastasis predictor. Eur J Cancer 39: 184-191.

12. Ahn HS, Shin YS, Park PJ, et al. (2012): Serum biomarker panels for the diagnosis of gastric adenocarcinoma. Br J Cancer 106: 733-739.

13. Baj-Krzyworzeka M, Weglarczyk K, Mytar B, et al. (2011): Tumour-derived microvesicles contain interleukin- 8 and modulate production of chemokines by human monocytes. Anticancer Res 31: 1329-1335.

14. Sugasawa H, Ichikura T, Tsujimoto H, et al. (2008): Prognostic significance of expression of CCL5/RANTES receptors in patients with gastric cancer. J Surg Oncol 97: 445-450.

15. Setoyama T, Arima H, Hokita S, Aikou T (2007): Clinical implication of CXCL12 expression in gastric cancer (SDF-1) Ann Surg Oncol 14: 3154-3158.

16. Soria G, Ben-Baruch A (2008): The inflammatory chemokines CCL2 and CCL5 in breast cancer. Cancer Lett 267: 271285.

17. Jianghong W, Xiaowen L, Yanong W (2013): Predictive value of preoperative serum CCL2, CCL18 and VEGF for the patients with gastric cancer. BMC Clin Pathol 22: 15-19.

18. Ohta M, Kitadai Y, Tanaka S, et al. (2003): Monocyte chemoattractant protein-1 expression correlates with macrophage infliltration and tumour vascularity in human gastric carcinomas. Int J Oncol 22: 773-778.

19. Saito S, Kitayama J, Jin ZX, et al. (2003): Beta-chemokine, macrophage inflammatory protein-1beta (MIP-1beta), is highly expressed in diffuse type human gastric cancers. J Exp Clin Cancer Res 22: 453-459.

20. Rajkumar T, Vijayalakshmi N, Gopal G, et al. (2010): Identification and validation of genes involved in gastric tumourigenesis. Cancer Cell Int 10: 45-57.

21. Kuai WX, Wang Q, Yang XZ, et al. (2012): Interleukin-8 associates with adhesion, migration, invasion and chemosensitivity of human gastric cancer cells. World J Gastroenterol 18: 979-985. 
22. Sarvaiya PJ, Guo D, Ulasov I, et al. (2013): Chemokines in tumour progression and metastasis. Oncotarget 4: 2171-2185.

23. Kim HJ, Song DE, Lim SY, et al. (2011): Loss of the promyelocytic leukemia protein in gastric cancer: implications for IP-10 expression and tumour-infiltrating lymphocytes. PLoS One 6: e26264.

24. Ohtani H, Jin Z, Takegawa S, et al. (2009): Abundant expression of CXCL9 (MIG) by stromal cells that include dendritic cells and accumulation of CXCR3+ T cells in lymphocyte-rich gastric carcinoma. J Pathol 217: 21-31.

25. Eck M, Schmausser B, Scheller K, et al. (2000): Pleiotropic effects of CXC chemokines in gastric carcinoma: differences in CXCL8 and CXCL1 expression between diffuse and intestinal types of gastric carcinoma. Clin Exp Immunol 122: 192-199. 